Original

\title{
Influences of differences in tray design and impression material on impression pressure at edentulous mandible
}

\author{
Sayumi Inoue, Misao Kawara, Takashi Iida, Masatoshi Iwasaki, and Osamu Komiyama \\ Department of Oral Function and Rehabilitation, Nihon University School of Dentistry at Matsudo, \\ Matsudo, Japan
}

(Received October 17, 2016; Accepted December 10, 2016)

\begin{abstract}
The aim of this study was to examine the effects of tray design and impression material on impression pressure in a clinical simulation model of an edentulous mandible. Two types of polyvinylsiloxane elastomer, one type of polyether elastomer, and one type of alginate were used. The three tray types had no relief, $0.36 \mathrm{~mm}$ of relief, or $1.4 \mathrm{~mm}$ of relief, with or without escape holes. Impression pressure was measured at the median alveolar crest, the bilateral alveolar crests corresponding to molars, and the bilateral buccal shelves. Impression pressure significantly differed in relation to tray design and sensor position. In trays without escape holes, impression pressure was highest at the median alveolar crest and lowest at the buccal shelves, for all impression materials. However, impression material had no significant effects on impression pressure. Our results suggest that bite-pressure load on alveolar crests can be alleviated by making an impression with a tray that has relief and escape holes, while applying pressure to buccal shelves and almost no pressure to alveolar crests.
\end{abstract}

Keywords: edentulous impression; impression pressure; tray design; selective pressure impression.

Correspondence to Dr. Takashi Iida, Department of Oral Function and Rehabilitation, Nihon University School of Dentistry at Matsudo, 2-870-1 Sakaecho-nishi, Matsudo, Chiba 271-8587, Japan

Fax:+81-47-360-9615 E-mail: iida.takashi96@nihon-u.ac.jp

J-STAGE Advance Publication: August 31, 2017

Color figures can be viewed in the online issue at J-STAGE.

doi.org/10.2334/josnusd.16-0731

DN/JST.JSTAGE/josnusd/16-0731

\section{Introduction}

In edentulous patients, progression of bone resorption is markedly faster in the mandible than in the maxilla, and diverse changes occur in the ridges of the mandible (1-3). One study reported that, in alveolar crests of edentulous mandibles, bone density tends to be lower when ridges are higher (4). To inhibit progression of bone resorption in the edentulous mandible, dentures should therefore be designed to minimize bite pressure on mandibular alveolar crests. Thus, pressure should not be applied to alveolar crests when making an impression. Because of considerations regarding bite pressure, the buccal shelves have been used as the main supporting areas in mandibular complete dentures (5-7).

Techniques for making impressions of edentulous ridges are classified as non-pressure, pressure, and selective pressure impressions (8-14). Non-pressure impression is a technique for making an impression of the stationary state of ridges while minimizing impression pressure. Pressure impression is used to make an impression of ridges under pressure and replicates conditions experienced during jaw functioning. Selective pressure impression requires adjustment of pressure applied to ridges, in accordance with the pressure-bearing capacity of each site. Pressure is adjusted by varying spacer thickness and the number and size of escape holes. Hyde et al. reported that dentures prepared using selective pressure technique were preferable to those prepared with conventional techniques (12). Some evidence suggests that the selective pressure technique is the best method for making impressions of edentulous ridges (9). These reports indicate that pressure applied to the interior of the tray at each site should be examined when making 


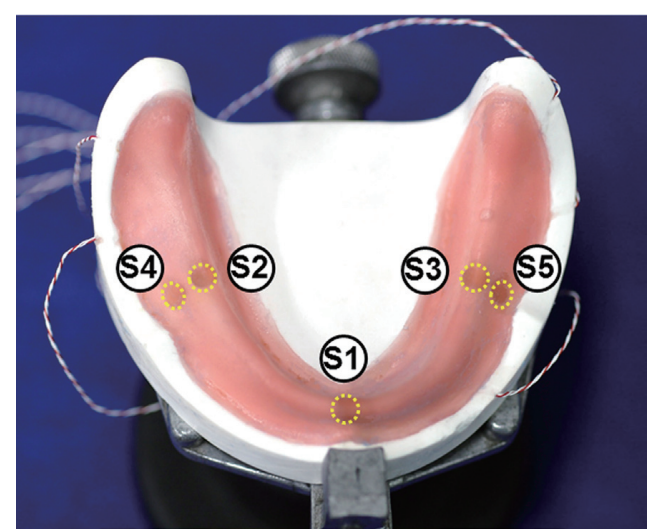

Fig. 1 Overview of sensor installation sites in a clinical simulation model of an edentulous mandible. S1, median alveolar crest; S2, right alveolar crest; S3, left alveolar crest; S4, right buccal shelf; S5, left buccal shelf.

Table 1 Summary of impression materials

\begin{tabular}{lll}
\hline Constituent & Material & Manufacturer \\
\hline Polyvinylsiloxane & Exadenture (ED) & GC, Tokyo, Japan \\
Polyvinylsiloxane & Affinis precious light body (AF) & YOSHIDA, Tokyo, Japan \\
Polyether & Impregum soft medium body (IG) & 3M ESPE, Neuss, Germany \\
Alginate & Aroma fine plus normal set (AR) & GC, Tokyo, Japan \\
\hline
\end{tabular}

impressions with selective pressure impression.

Several studies have investigated impression pressure in edentulous jaws (15-20). Frank et al. examined impression pressure in the edentulous maxilla and reported that it varies in relation to tray design (15). Iwasaki et al. used a clinical simulation model of an edentulous maxilla to investigate the effect of relief thickness on impression pressure and found that impression pressure in the tray was equalized by relief (20). Other studies found that tray design did not greatly affect impression pressure in the tray when making impressions $(17,19)$. These reports suggest that clinicians can select sites that do and do not receive pressure when making impressions for complete dentures. However, specific procedures have not been established for selective pressure impression and tray design when making impressions for complete dentures. In particular, few studies have examined impression pressure applied to alveolar crests and buccal shelves in an edentulous mandible (19).

In this study, we examined the effects of tray design and impression material on impression pressure in an edentulous mandible model that simulates displaceability. Our aim was to identify the optimal selective pressure impression technique for the edentulous mandible.

\section{Simulation model}

\section{Materials and Methods}

We used an existing plaster model (MAP-34, NISSIN, Kyoto, Japan) as a model of an edentulous mandible.
After grinding the surface of the plaster model by the amount of mucosal thickness $(2 \mathrm{~mm}$ at the alveolar crests and $1.5 \mathrm{~mm}$ at other locations) (21), we embedded small pressure sensors (PSM-2KAB, Kyowa Electronic Instruments Co., Ltd., Tokyo, Japan; diameter $3.5 \mathrm{~mm}$; thickness $0.65 \mathrm{~mm}$ ) at five locations: the median alveolar crest (S1), the bilateral alveolar crests corresponding to the molars (right side S2, left side S3), and the bilateral buccal shelves (right side S4, left side S5) (Fig. 1). For the pseudomucosa, we used denture soft lining materials for removable dentures (Sofreliner Tough Supersoft, Tokuyama Dental, Tokyo, Japan).

\section{Impression materials}

Two types of polyvinylsiloxane elastomer, one type of polyether elastomer (polyether elastomeric impression material), and one type of alginate were used. As was the case in a study by Kawara et al., the two types of polyvinylsiloxane selected were Exadenture (ED) with a moderate storage modulus and Affinis precious light body (AF) with the lowest storage modulus (22). Impregum soft medium body (IG) was selected as the polyether rubber impression material, and Aroma fine plus normal set (AR) was used as the alginate (Table 1). Mixing was carried out in accordance with manufacturer instructions.

\section{Trays}

Using autopolymerizing acrylic resin (Ostron II, GC, Tokyo, Japan), we prepared six types of trays, with 

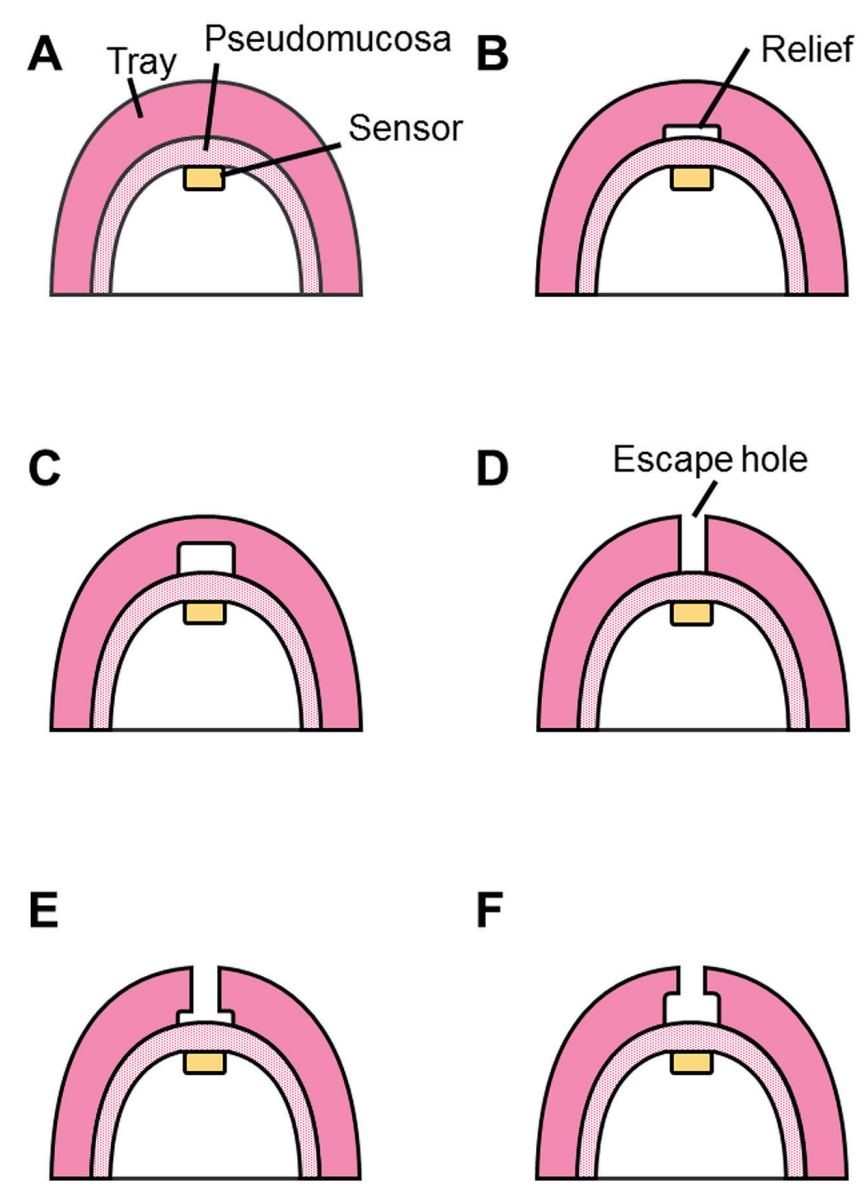

Fig. 2 Sectional views of tray designs R0 (A), R0.36 (B), R1.4 (C), R0V (D), R0.36V (E), and R1.4V (F).

different relief and escape hole conditions. Relief was provided at the alveolar crests so as to cover the anterior teeth at a width of $4 \mathrm{~mm}$ and the molars at a width of $6 \mathrm{~mm}$. The relief conditions were no relief (R0), 0.36 $\mathrm{mm}$ of relief using sheet wax (R0.36), and $1.4 \mathrm{~mm}$ of relief using paraffin wax (R1.4). Escape holes (diameter $2.3 \mathrm{~mm}$ ) were provided by using a round bar in the trays produced under the three relief conditions. Two escape hole conditions were used: escape holes at locations corresponding to S1, S2, and S3 (V) or no escape holes. Measurements were collected for a total of six tray conditions, namely, the three relief conditions and two escape hole conditions (Fig. 2).

\section{Measurement method}

For the loading device, in accordance with the method of Iwasaki et al. we used a dental surveyor (J.M. Ney Company, Bloomfield, CT, USA) and a model table, along with a metal indenter that provided a site for placing a 2-kg weight (20). Mixing was performed at room temperature $\left(23 \pm 1^{\circ} \mathrm{C}\right)$ and a humidity of $50 \pm 5 \%$.
A total of 24 conditions comprising the four impression materials and six tray conditions were each measured 5 times. The mixing time and operation time for each of the impression materials was set at a total of $60 \mathrm{~s}$, and seating was begun immediately after mixing. The impression material was injected into the tray, to keep it as uniform as possible. In accordance with a report by Komiyama et al., impression pressure was defined as the pressure at each measurement site $120 \mathrm{~s}$ after the start of seating (18).

\section{Data analysis}

The measurements from the pressure sensors were saved on a personal computer at a sampling rate of $10 \mathrm{~Hz}$ by means of a sensor interface (PCD-300A, Kyowa Electronic Instruments Co., Ltd.). The pressure $(\mathrm{kPa})$ at each sensor was determined by using a conversion formula. For each impression material, the measurement was performed 5 times for each of the six types of tray, and the mean values were determined. For each impression material, the various pressure sensor values measured $120 \mathrm{~s}$ after the start of measurement were compared.

In statistical analysis, the assumption of normality was tested with the Shapiro-Wilk statistic, and the homogeneity of variance was tested with the Levene test before analysis of variance (ANOVA). Impression pressures in each measurement were analyzed by using three-way ANOVA with impression material, tray design, and sensor position as analytical factors. Simple main effect and multiple comparisons (Scheffé's test) were used to test the difference between values by analysis of the three-way interaction effect. The significance level was defined as 5\%. All statistical analysis was performed by using Dr. SPSS II for Windows (IBM, Tokyo, Japan).

\section{Results}

Table 2 shows impression pressures for each factor in three-way ANOVA. Tray design and sensor position were significantly associated with impression pressure $(P<0.05)$ (Table 2). There were significant three-way interactions between sensor, impression material, and tray. Differences were analyzed by using simple main effect and multiple comparisons (Scheffé's test).

\section{ED}

Figure 3A shows impression pressures at each sensor in ED. In the R0 and R0.36 trays, the highest impression pressure was at $\mathrm{S} 1$ and the lowest impression pressures were at S4 and S5. No significant difference in impression pressure was seen in any comparison of sensors in the R1.4 tray. In the R0V, R0.36V, and R1.4V trays, 
Table 2 Comparison of sources and interactions between sources

\begin{tabular}{llllll}
\hline Source & Type III sum of square & Degrees of freedom & Mean square & F value & $P$ value \\
\hline Sensor & 4448.975 & 4 & 1112.244 & 80.712 & 0.000 \\
Impression material & 94.330 & 3 & 31.443 & 2.282 & 0.078 \\
Tray & 9577.571 & 5 & 1915.514 & 139.003 & 0.000 \\
Sensor*Impression material & 2613.942 & 12 & 217.829 & 15.807 & 0.000 \\
Sensor*Tray & 42886.056 & 20 & 2144.303 & 155.605 & 0.000 \\
Impression material*Tray & 1600.260 & 15 & 106.684 & 7.742 & 0.000 \\
Sensor*Impression material*Tray & 3506.049 & 60 & 58.434 & 4.240 & 0.000 \\
Model & $64727.183^{\mathrm{a}}$ & 119 & 543.926 & 39.471 & 0.000 \\
Error & 6614.593 & 480 & 13.780 & & \\
\hline
\end{tabular}

${ }^{\mathrm{a}} R^{2}=0.907$

\section{A}

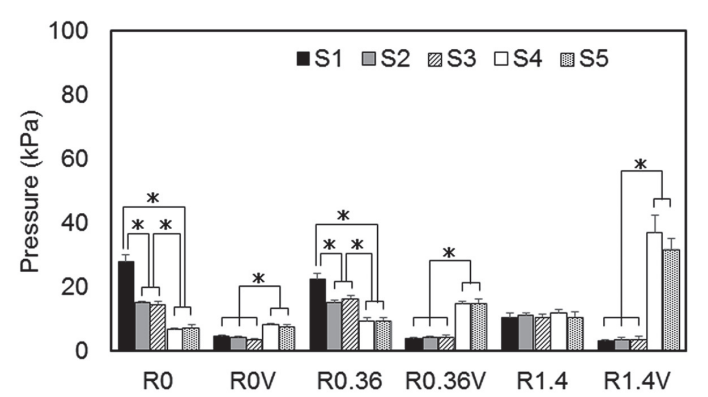

C

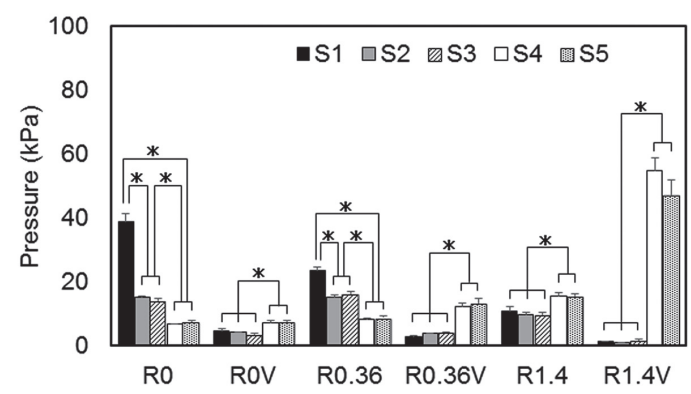

B

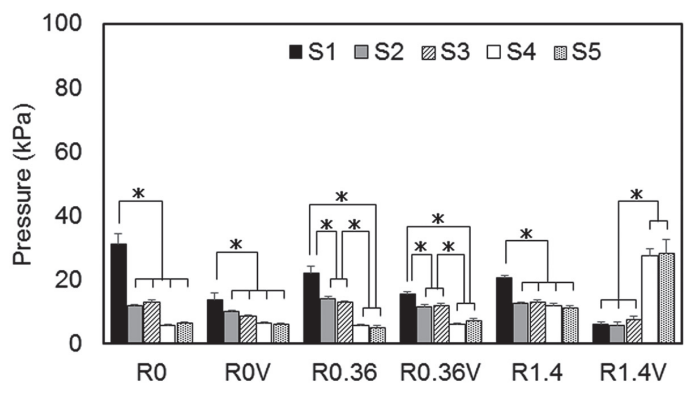

D

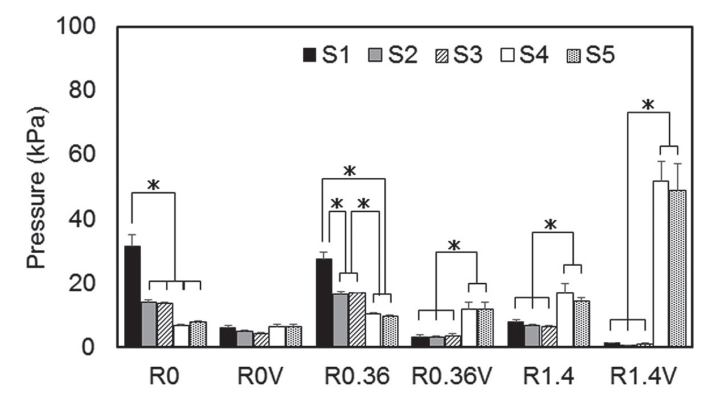

Fig. 3 Pressure at sensors in Exadenture (A), Affinis precious light body (B), Impregum soft medium body (C), and Aroma fine plus normal set (D). S1, median alveolar crest; S2, right alveolar crest; S3, left alveolar crest; S4, right buccal shelf; $\mathrm{S} 5$, left buccal shelf; $\mathrm{R}$, relief; $\mathrm{V}$, escape hole. ${ }^{*} P<0.05$.

impression pressures at $\mathrm{S} 4$ and $\mathrm{S} 5$ were significantly higher than those at $\mathrm{S} 1, \mathrm{~S} 2$, and $\mathrm{S} 3(P<0.05)$.

\section{AF}

Figure 3B shows impression pressures at each sensor in AF. In the R0, R0.36, and R1.4 trays, the impression pressure at $\mathrm{S} 1$ was significantly higher than those at the other sensors $(P<0.05)$. In the R0V and R0.36V trays, the impression pressure at $\mathrm{S} 1$ was significantly higher than those at the other sensors $(P<0.05)$. In the R1.4V tray, the impression pressures at $\mathrm{S} 4$ and $\mathrm{S} 5$ were significantly higher than those at $\mathrm{S} 1, \mathrm{~S} 2$, and $\mathrm{S} 3(P<0.05)$.

\section{IG}

Figure 3C shows impression pressures at each sensor in
IG. In the R0 and R0.36 trays, impression pressure was highest at S1 and lowest at S4 and S5. In the R1.4 trays, the impression pressures at $\mathrm{S} 4$ and $\mathrm{S} 5$ were significantly higher than those at $\mathrm{S} 1, \mathrm{~S} 2$, and $\mathrm{S} 3(P<0.05)$. In the $\mathrm{R} 0 \mathrm{~V}, \mathrm{R} 0.36 \mathrm{~V}$, and $\mathrm{R} 1.4 \mathrm{~V}$ trays, the impression pressures at $\mathrm{S} 4$ and $\mathrm{S} 5$ were significantly higher than those at $\mathrm{S} 1$, $\mathrm{S} 2$, and $\mathrm{S} 3(P<0.05)$.

\section{AR}

Figure 3D shows impression pressures at each sensor in AR. In the R0 and R0.36 trays, impression pressure was highest at S1 and lowest at S4 and S5. In the R1.4 trays, the impression pressures at $\mathrm{S} 4$ and $\mathrm{S} 5$ were significantly higher than those at S1, S2, and S3 $(P<0.05)$. Impression pressures in the R0V tray did not significantly differ. 
In the R0.36V and R1.4V trays, impression pressures at $\mathrm{S} 4$ and S5 were significantly higher than those at S1, S2, and $\mathrm{S} 3(P<0.05)$.

\section{Discussion}

Impression pressures at the various sensors did not seem to be affected by impression material. In trays without relief or escape holes (R0), impression pressure was highest at the median alveolar crest and lowest at the buccal shelves, in all impression materials. In R0V trays with escape holes, impression pressure tended to be more equal than in the R0 trays, but impression pressure applied to the buccal shelves did not change. In trays with $0.36 \mathrm{~mm}$ of relief (R0.36), there was no real effect on impression pressure within the tray, but when escape holes were also present (R0.36V), impression pressure applied to the buccal shelves was higher than that applied to alveolar crests, in ED, IG, and AR. In trays with 1.4 $\mathrm{mm}$ of relief (R1.4), impression pressure applied to the buccal shelves was higher than that applied to alveolar crests, in IG and AR. Furthermore, in trays with $1.4 \mathrm{~mm}$ of relief and escape holes (R1.4V), impression pressure applied to buccal shelves was markedly higher than that applied to alveolar crests, in all impression materials. The effect on impression pressure was greater when the relief thickness was $1.4 \mathrm{~mm}$ rather than $0.36 \mathrm{~mm}$ and was markedly greater in trays with escape holes than in those without escape holes.

In the edentulous maxilla, the highest impression pressure when making impressions is applied at the center $(16,18)$, most likely because impression pressure is concentrated at the center of the tray, where the outflow region of the impression material is small. It is also believed that in the mandible, impression pressure is similarly concentrated at the median alveolar crest. Ahmad et al. used an edentulous mandible model to examine the effects of tray design and impression material on impression pressure at the buccal shelves and median alveolar crest and found that impression pressure was higher at the median alveolar crest than at the buccal shelves (19). Our results also show that the highest impression pressure in $\mathrm{R} 0$ was at the median alveolar crest and that the lowest impression pressure in $\mathrm{R} 0$ was at the buccal shelves, in all impression materials. Our findings also suggest that since the alveolar crest is narrow and the area of the alveolar crest is small for an anatomical structure, impression pressure per unit area at the median alveolar crest was higher than that at the buccal shelves in the mandible.

Iwasaki et al. used a model of an edentulous maxilla to study impression pressure dynamics within trays with different relief thicknesses and reported that impression pressure was equalized by providing a relief space (20). In an examination of the effects of escape holes and relief spaces on making edentulous maxillary impressions, Komiyama et al. reported that when escape holes and relief were not provided, impression pressure was higher at the palate than at the alveolar crests; however, when escape holes at the mid-palatal suture and relief were provided, this effect was reversed and impression pressure was higher at the alveolar crests than at the palate (18). The present results showed that impression pressure on buccal shelves in trays with $1.4 \mathrm{~mm}$ of relief and escape holes at the alveolar crests (R1.4V) was markedly higher than that on the alveolar crests, in all impression materials. Our findings suggest that by increasing relief thickness, the space through which the impression material flows near the mandibular alveolar crests increases and impression pressure at the mandibular alveolar crests decreases. In addition, the present study shows that impression pressure on alveolar crests was lower for trays with escape holes than for those without escape holes, and that impression pressure on the shelves was greater for trays with escape holes than for those without escape holes. Our findings suggest that, in the mandible, impression pressure can also be simultaneously applied to the buccal shelves by providing relief and escape holes.

A previous study reported that impression pressure varies in relation to the type of impression material (19). Ahmad et al. found that low-viscosity impression materials are suitable for making impressions of the edentulous mandible (19). However, they provided relief on the entire mandible, excluding the buccal frenum and retromolar pad, and the positional relationships between the impression pressure transducers and escape holes differed from those in the present study (19). The present study showed that although tray design and sensor positions had significant effects on impression pressure, impression material had no significant effects. Iwasaki et al. studied impression pressure in the edentulous maxilla and reported that relief with an impression material with a low storage elastic modulus had a greater cushioning effect than relief with an impression material with a high storage elastic modulus (20). The focus of the present study was impression pressure in the edentulous mandible, and we believe that the effect of tray configuration was greater than that of impression material because the tray area is smaller for the mandible than for the maxilla. However, we noted significant interactions between sensor, impression material, and tray in this study. Future studies should investigate the effects of differences in impression material on impression pressure 
in a clinical simulation model of an edentulous mandible, to establish a selective pressure impression technique for an edentulous mandible.

The present study used a clinical simulation model of an edentulous mandible, but mandibular ridge configurations are not clinically uniform. In addition, we believe that studying impression pressure in relation to the diameter of escape holes will lead to development of a better selective pressure impression technique.

Our findings suggest that use of a tray with relief and escape holes for selective pressure impression of an edentulous mandible, regardless of the type of impression material, results in a pressure distribution in which impression pressure at the alveolar crests is decreased and bite pressure is carried by the buccal shelves. These results are useful for impression techniques that consider inhibition of bone resorption progression in edentulous mandibular ridges.

In conclusion, when making impressions of an edentulous mandible, bite pressure on alveolar crests can be alleviated by making an impression with a tray with both relief and escape holes while applying pressure to the buccal shelves and applying almost no pressure to the alveolar crests. These characteristics will suppress ridge resorption in alveolar crests.

\section{Acknowledgments}

This study was supported by a Grant-in-Aid for Young Scientists (B26861655) from the Ministry of Education, Culture, Sports, Science and Technology of Japan, and a Grant-in-Aid for Scientific Research (C25463027 and C26462959) from the Japan Society for the Promotion of Science.

\section{Conflict of interest}

None declared.

\section{References}

1. Atwood DA (1971) Reduction of residual ridges: a major oral disease entity. J Prosthet Dent 26, 266-279.

2. Tallgren A (1972) The continuing reduction of the residual alveolar ridges in complete denture wearers: a mixed-longitudinal study covering 25 years. J Prosthet Dent 27, 120-132.

3. Kingsmill VJ (1999) Post-extraction remodeling of the adult mandible. Crit Rev Oral Biol Med 10, 384-404.

4. Inoue S, Kawara M, Iida T, Iwasaki M, Komiyama O, Kaneda $\mathrm{T}$ (2016) Analysis of correlation between height of residual ridge and bone density of residual ridge crest at edentulous mandible using computed tomography. J Prosthodont Res, doi: 10.1016/j.jpor.2016.09.003.

5. Boucher CO (1951) A critical analysis of mid-century impression techniques for full dentures. J Prosthet Dent 1, 472-491.

6. Henry AC (1965) Complete denture impressions. J Prosthet
Dent 15, 603-614.

7. Tan KM, Singer MT, Masri R, Driscoll CF (2009) Modified fluid wax impression for a severely resorbed edentulous mandibular ridge. J Prosthet Dent 101, 279-282.

8. el-Khodary NM, Shaaban NA, Abdel-Hakim AM (1985) Effect of complete denture impression technique on the oral mucosa. J Prosthet Dent 53, 543-549.

9. Weng BX, Khlevnoy V (1995) Pressure control for complete denture impressions. Oral Health 85, 21-27.

10. Petropoulos VC, Rashedi B (2003) Current concepts and techniques in complete denture final impression procedures. $\mathrm{J}$ Prosthodont 12, 280-287.

11. Duncan JP, Raghavendra S, Taylor TD (2004) A selectivepressure impression technique for the edentulous maxilla. $\mathrm{J}$ Prosthet Dent 92, 299-301.

12. Hyde TP, Craddock HL, Blance A, Brunton PA (2010) A cross-over randomised controlled trial of selective pressure impressions for lower complete dentures. J Dent 38, 853-858.

13. Gupta A, Singhal P, Negi P (2014) Selective pressure impression technique: an overview. J Evol Med Dent Sci 3, 8110-8114.

14. Shah RJ, Lagdive SB, Barajod PK, Patel MN (2015) Complete denture impression procedures and techniques practiced by dentists across the state of Gujarat: a survey. IOSR-JDMS 14, $1-11$.

15. Frank RP (1969) Analysis of pressures produced during maxillary edentulous impression procedures. J Prosthet Dent 22, 400-413.

16. Rihani A (1981) Pressures involved in making upper edentulous impressions. J Prosthet Dent 46, 610-614.

17. Masri R, Driscoll CF, Burkhardt J, von Fraunhofer A, Romberg E (2002) Pressure generated on a simulated oral analog by impression materials in custom trays of different designs. J Prosthodont 11, 155-160.

18. Komiyama O, Saeki H, Kawara M, Kobayashi K, Otake S (2004) Effects of relief space and escape holes on pressure characteristics of maxillary edentulous impressions. J Prosthet Dent 91, 570-576.

19. Al-Ahmad A, Masri R, Driscoll CF, von Fraunhofer J, Romberg E (2006) Pressure generated on a simulated mandibular oral analog by impression materials in custom trays of different design. J Prosthodont 15, 95-101.

20. Iwasaki M, Kawara M, Inoue S, Komiyama O, Iida T, Asano $\mathrm{T}$ (2016) Pressure dynamics in the trays caused by differences of the various impression materials and thickness of the relief in the maxillary edentulous model. J Prosthodont Res 60, 123-130.

21. Dong J, Zhang FY, Wu GH, Zhang W, Yin J (2015) Measurement of mucosal thickness in denture-bearing area of edentulous mandible. Chin Med J 128, 342-347.

22. Kawara M, Iwasaki M, Iwata $\mathrm{Y}$, Komoda $\mathrm{Y}$, Inoue $\mathrm{S}$, Komiyama O et al. (2015) Rheological properties of elastomeric impression materials for selective pressure impression technique. J Prosthodont Res 59, 254-261. 